\title{
Electromagnetically Induced Transparency and Light Storage in an Atomic Mott Insulator
}

\author{
U. Schnorrberger ${ }^{1}$, J. D. Thompson ${ }^{1,2}$, S. Trotzky ${ }^{1}$, R. Pugatch ${ }^{3}$, N. Davidson ${ }^{3}$, S. Kuhr ${ }^{1}$, and I. Bloch ${ }^{1,4}$ \\ ${ }^{1}$ Johannes Gutenberg-Universität, Institut für Physik, Staudingerweg 7, 55128 Mainz, Germany \\ ${ }^{2}$ Department of Physics, Harvard University, Cambridge, Massachusetts 02138, USA \\ ${ }^{3}$ Department of Physics of Complex Systems, Weizmann Institute of Science, Rehovot 76100, Israel \\ ${ }^{4}$ Max-Planck-Institut für Quantenoptik, Hans-Kopfermann-Str. 1, 85748 Garching, Germany
}

(Dated: March 1, 2009)

\begin{abstract}
We experimentally demonstrate electromagnetically induced transparency and light storage with ultracold ${ }^{87} \mathrm{Rb}$ atoms in a Mott insulating state in a three dimensional optical lattice. We have observed light storage times of $\simeq 240 \mathrm{~ms}$, to our knowledge the longest ever achieved in ultracold atomic samples. Using the differential light shift caused by a spatially inhomogeneous far detuned light field we imprint a "phase gradient" across the atomic sample, resulting in controlled angular redirection of the retrieved light pulse.
\end{abstract}

PACS numbers: $37.10 . J k, 42.50 . G y$

Coherent interaction between light and matter plays an important role in many quantum information and quantum communication schemes [1, 2]. In particular, it is desirable to transfer quantum states from photonic, "flying" qubits to matter-based systems for storage and processing [3]. In this context, electromagnetically induced transparency (EIT) has proven extremely useful, since it allows an incoming light pulse to be converted into a stationary superposition of internal states and back into a light pulse [4, 5, [6, 7]. This effect has successfully been used to map quantum states of light onto cold atomic ensembles [8] or even to transmit quantum information between two such remote quantum memories [9]. EIT and light storage have been realized in crystals 10], atomic vapors [7, 11] and in ultracold atomic ensembles $[6,12,13]$. In crystals, storage times of several seconds have been achieved [14]. In vapor cells, inelastic collisions with other atoms or with the walls usually limit the coherence times to a few milliseconds [15, 16]. In cold atomic samples the light storage times are also on a millisecond timescale [6]. Using magnetically insensitive states, storage times of up to $6 \mathrm{~ms}$ were recently observed even for single quantum excitations in cold atomic gases, limited by loss of atoms [17] or thermal diffusion [18].

Ultracold atoms in a Mott insulator (MI) state with unity filling in a deep 3D optical lattice are ideal for light storage, as they experience no diffusion and no collisional interaction. In the present work, we demonstrate EIT and long light storage in such an environment. The minimal dephasing observed allows for many possibilities for processing stored information using advanced manipulation techniques for atomic many-body states in optical lattices (see Ref. [19] and references therein). Light pulses can be stored in an atomic spin wave in the MI, transformed, and then efficiently mapped back into photonic modes. As an example of such a spin-wave manipulation, we imprint a "phase gradient" across the atomic sample using a spatially varying differential light shift of the two ground state levels. This spatial phase gradient results in a controlled change of the direction of the

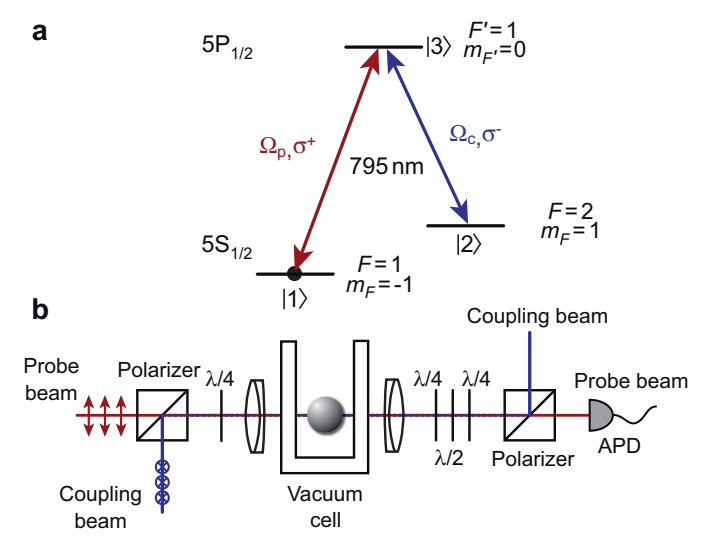

FIG. 1: (a) EIT $\Lambda$-system in ${ }^{87} \mathrm{Rb}$ ( $\mathrm{D}_{1}$-line). The transition between the two ground states $|1\rangle$ and $|2\rangle$ is insensitive to magnetic field fluctuations to first order at $B \simeq 3.23$ G. (b) Experimental setup. Probe and coupling beam are used in a collinear configuration and are focussed onto the atoms in the optical lattice.

restored pulse. By controlling non-classical atomic spin excitations, atoms in optical lattices could even be turned into novel non-classical light sources [20, 21] or lead to deterministic photonic phase gates at the single photon level [3].

In our experiment we begin with ultracold ${ }^{87} \mathrm{Rb}$ atoms in the $\left|F=1, m_{F}=-1\right\rangle \equiv|1\rangle$ state in an optical lattice consisting of three mutually orthogonal retroreflected laser beams each with $1 / e^{2}$-radius $\approx 150 \mu \mathrm{m}$. Two of the lattice beams are red detuned $\left(\lambda_{y, z}=844 \mathrm{~nm}\right)$, while the third is blue detuned $\left(\lambda_{x}=765 \mathrm{~nm}\right)$. In a sufficiently deep lattice (30 $E_{r} ; E_{r}=h^{2} / 2 m \lambda_{z}^{2}$ is the recoil energy), the many-body ground state is a MI with a well-defined number of atoms on each lattice site.

For EIT, we use a $\Lambda$-system consisting of the two Zeeman sublevels $|1\rangle$ and $\left|F=2, m_{F}=+1\right\rangle \equiv|2\rangle$ of the $5 \mathrm{~S}_{1 / 2}$ ground state, and the $\left|F^{\prime}=1, m_{F}=0\right\rangle \equiv|3\rangle$ level of the $5 \mathrm{P}_{1 / 2}$ excited state [Fig. 1(a)]. At a field of 


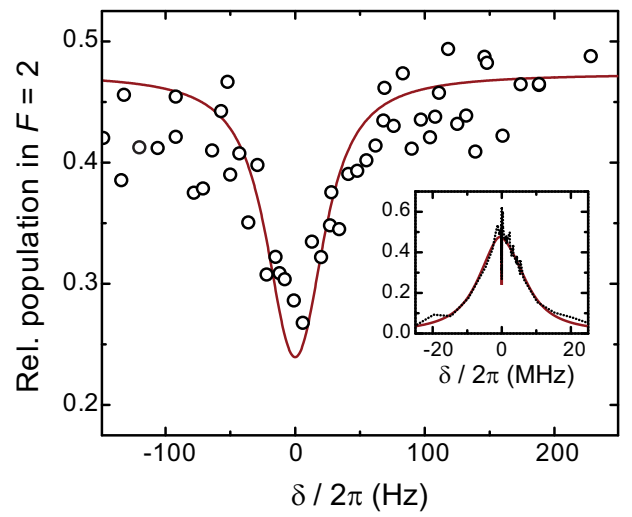

FIG. 2: Observation of EIT in a Mott insulator. Shown is the fraction of atoms transferred from $F=1$ to $F=2$ by a $200 \mathrm{~ms}$ probe laser pulse, as a function of the two-photon detuning $\delta=\omega_{p}-\omega_{c}-\omega_{21}$. The observed EIT window has a width of $81(10) \mathrm{Hz}$. The inset shows the total lineshape. The red line is a prediction from a rate equation model (see text).

$B \simeq 3.23 \mathrm{G}$, the states $|1\rangle$ and $|2\rangle$ have the same firstorder Zeeman shift [22]. The coupling laser light with Rabi frequency $\Omega_{c}$ is $\sigma^{-}$-polarized and is resonant with the $|2\rangle \leftrightarrow|3\rangle$ transition. The probe laser (Rabi frequency $\Omega_{p}$, frequency $\omega_{p}, \sigma^{+}$-pol.) is phase locked to the coupling laser with a difference frequency corresponding to the ground state hyperfine splitting. We use a collinear arrangement of probe- and coupling beams [Fig. 1(b)] in order to avoid momentum transfer to the atoms. The two beams are overlapped on a Glan-Thompson polarizer before a $\lambda / 4$ waveplate converts the linear into circular polarizations. A lens system focusses the beams onto the atomic sample. The coupling beam has a $1 / \mathrm{e}^{2}-$ radius of $\simeq 150 \mu \mathrm{m}$, much larger than the diameter of the atomic sample (typically $26 \mu \mathrm{m}$ ), in order to facilitate the alignment and to create a spatially homogeneous coupling laser field. The probe laser beam has a radius of $\simeq 40 \mu \mathrm{m}$. The outgoing beams are separated using polarization optics (suppression ratios of $10^{3}-10^{4}$ ) and the probe beam is directed onto an avalanche photodiode (APD, Analog Modules 712A-4).

We first observe EIT, in particular the existence of a narrow transmission window, in an atomic sample of $\simeq 9 \times 10^{4}$ atoms, which in our system corresponds to a MI with only singly occupied sites. The atomic sample is an ellipsoid with radii $r_{x}=8.6 \mu \mathrm{m}$ and $r_{y, z}=13.1 \mu \mathrm{m}$. We shine in the coupling laser $\left(\Omega_{c}=2 \pi \times 26(5) \mathrm{kHz}\right)$ and a weak probe laser pulse $\left(\Omega_{p}=2 \pi \times 7(2) \mathrm{kHz}\right)$ for $200 \mathrm{~ms}$. Due to the small system size and low powers necessary to achieve such a narrow EIT window, a direct measurement of probe transmission through the atom cloud is difficult in our case. Instead, we measure the fraction of atoms transferred by the probe laser to the $F=2$ manifold. We first detect the number of atoms $N_{2}$ in $F=2$ by resonant absorption imaging. A second image is taken $500 \mu$ s later with a repumper in order to also detect the atoms in the $F=1$ manifold $\left(N=N_{2}+N_{1}\right)$. The graphs in Fig. 2 show the relative population transfer $N_{2} / N$ as a function of the two-photon detuning. We observe an EIT transmission window (81(10) Hz FWHM) at the center of the absorption line. We calculate the fraction of atoms pumped from $|1\rangle$ into the $F=2$ manifold by a rate equation model. It includes the analytic expression for the linear susceptibility given in Ref. [4] and also accounts for the inhomogeneous optical depth (OD), which arises from the ellipsoidal cloud shape. To explain the observed population transfer to $F=2$ also at the center of the EIT window, we include a decay rate of the $|1\rangle-|2\rangle$ coherence $\gamma_{21}=2 \pi \times 10 \mathrm{~Hz}$ and transfer to $F=2$ by a fraction of $\pi$-polarized probe laser light $\left(\Omega_{p}^{\pi}\right)$ on the $|1\rangle \rightarrow\left|F^{\prime}=1, m_{F}=-1\right\rangle$ transition. The best agreement with the data is obtained with $\Omega_{c}=2 \pi \times 27 \mathrm{kHz}$, $\Omega_{p}=2 \pi \times 3.9 \mathrm{kHz}$ and $\Omega_{p}^{\pi}=0.2 \Omega_{p}$ (red line in Fig. 2), which are close to the measured values.

As a second experiment, we demonstrate the storage of light pulses (Fig. 3). After turning on the coupling beam, we apply a Gaussian-shaped probe pulse with $2.8 \mu \mathrm{s}$ FWHM. At the peak of this pulse, we shut off the probe and coupling beams simultaneously, within less than 50 ns. After waiting for a variable storage time, we turn on the coupling beam again and monitor the restored probe pulse on an APD. The second, retrieved pulse is much smaller than the first, incident pulse. From the ratio of their areas, we estimate the storage efficiency to be $3 \%$ for a large thermal cloud [Fig. 3(a)] and $0.3 \%$ for the MI. The small efficiency is partly caused by the mismatch of the size of the probe beam and the atomic
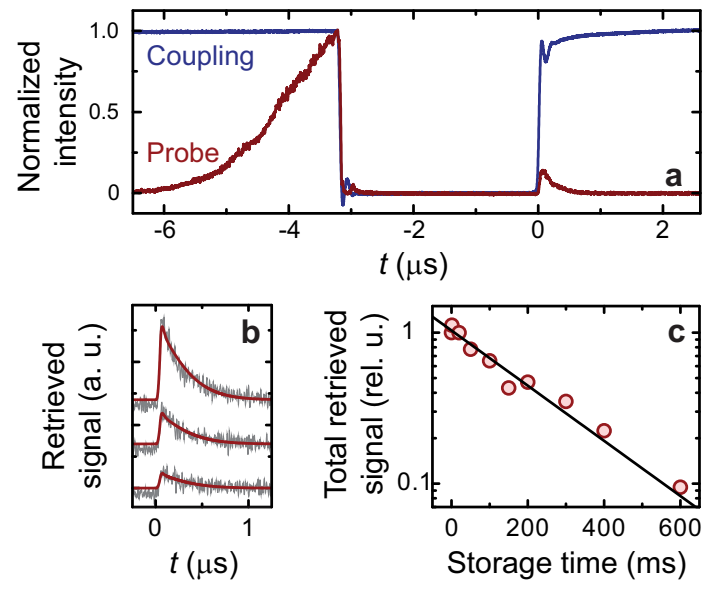

FIG. 3: Light storage. (a) Intensity of coupling (probe) beams, recorded on a photodiode before (after) the atomic sample during a light storage experiment with $3 \mu$ s storage time in a thermal cloud $\left(\Omega_{c}=2 \pi \times 4.9 \mathrm{MHz}, \Omega_{p}=\right.$ $2 \pi \times 920 \mathrm{kHz}, \simeq 10^{6}$ atoms) (b) Retrieved pulses for storage times of $t_{S}=1 \mathrm{~ms}, 200 \mathrm{~ms}, 400 \mathrm{~ms}$ (from top to bottom) in a Mott insulator. Traces are offset for better visibility $\left(\Omega_{c}=2 \pi \times 4.5 \mathrm{MHz}, \Omega_{p}=2 \pi \times 1.5 \mathrm{MHz}, \simeq 9 \times 10^{4}\right.$ atoms $)$. (c) Retrieved signal (relative to the signal at $t_{S}=3 \mu \mathrm{s}$ ) as a function of $t_{S}$. The line is an exponential fit with decay time $\tau=238(20) \mathrm{ms}$. 
sample (18\% geometrical overlap for the MI), leakage of the probe beam due to the finite OD of the sample (peak OD $\alpha=6.3$, see definition in Ref. [2]), and due to spontaneous emission during writing and retrieval phases. From numerical simulations based on the equations in [23], we estimate the efficiencies due to leakage and spontaneous emission as $11 \%$ for short storage times ( $3 \mu \mathrm{s})$. The same simulations were used to reproduce the retrieved pulse shapes shown in Fig. 3(b) with no free parameters other than the amplitude. Not included in the simulations are effects due to imperfect polarizations of probe and coupling beams.

We use the energy (integrated intensity) of the restored pulse as a measure of the stored light signal. As shown in Fig. 3 (c), fitting an exponential decay to the retrieved pulse power as a function of storage time yields a decay time constant of $\tau=238(20) \mathrm{ms}$. To independently measure the coherence time of the $|1\rangle+|2\rangle$ superposition, we performed a Ramsey experiment on the same states using an $\mathrm{rf}+$ microwave two-photon transition [22]. The visibility of the Ramsey fringes decays with a time constant of $2 \tau=436(22) \mathrm{ms}$, indicating that the decay of the stored light pulse is not caused by residual coupling light present during the storage time. The factor of two arises since the Ramsey fringe contrast measures the decay of the quantum amplitude coherence [24], whereas in the EIT signal we measure an intensity. A $\pi$-echo pulse does not restore the Ramsey signal contrast, so the decay time has to be attributed to an irreversible dephasing mechanism. We have ruled out magnetic field noise by measuring coherence times away from the "magic" field at $3.23 \mathrm{G}$. The coherence times are nearly unchanged at $6 \mathrm{G}$ and $2 \mathrm{G}$, where the differential shift of the $|1\rangle \leftrightarrow|2\rangle$ transition is at least an order of magnitude more sensitive to magnetic field fluctuations. We measured the coherence time vs lattice depth and found a maximum at $30-40 E_{r}$ for our experimental parameters. This indicates that the source of the coherence decay is due to heating in the optical lattice and to finite tunneling. The latter leads to an increased probability of having more than one atom per lattice site. In this case the interaction energy in the doubly occupied sites leads to an onsite dephasing with respect to the singly occupied sites. Increasing the lattice depth improves the coherence times due to the suppression of tunneling, but in turn the heating due to spontaneous light scattering and technical noise increases. Our analysis suggests that by simply using farther detuned lattices, even longer light storage times can be achieved.

In a non-collinear geometry, the difference in the wavevectors of coupling and probe beams, $\mathbf{k}_{c}-\mathbf{k}_{p}$, is stored as a spatial gradient in the phase of the atomic superposition state [6]:

$$
|D\rangle=\frac{\Omega_{c}|1\rangle-\Omega_{p} e^{i\left(\mathbf{k}_{c}-\mathbf{k}_{p}\right) \mathbf{r}}|2\rangle}{\sqrt{\Omega_{c}^{2}+\Omega_{p}^{2}}} .
$$

Here we reverse the logic leading to Eq. (11), and show

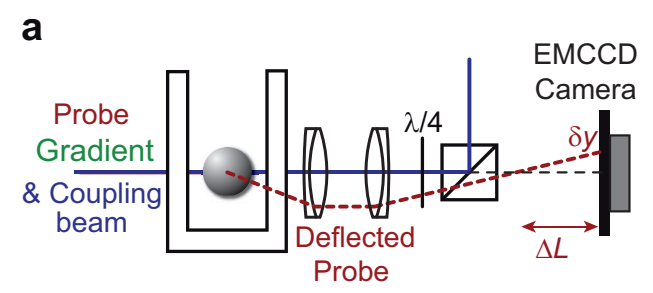

b
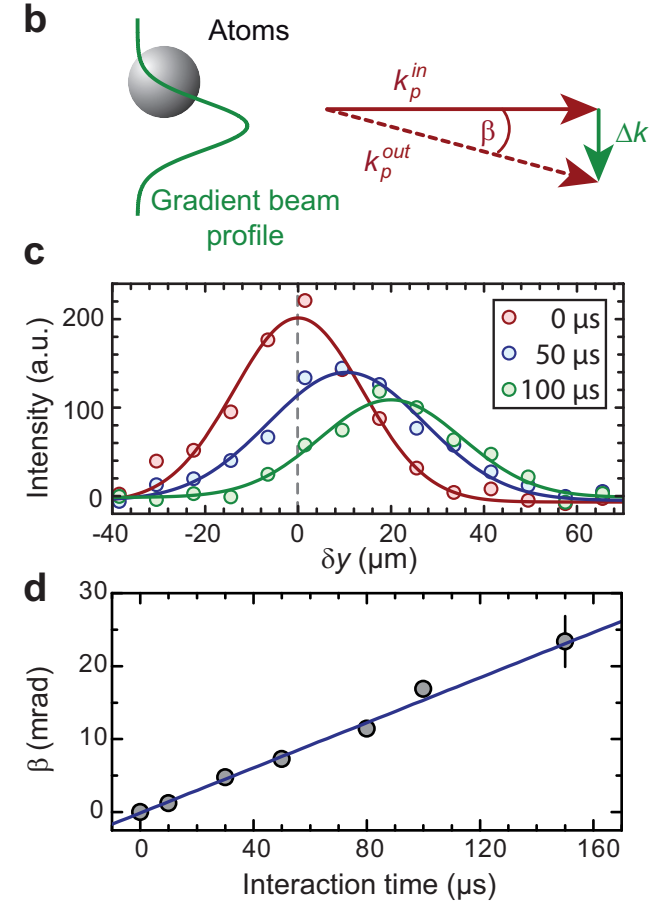

FIG. 4: Angular deflection of a stored light pulse. (a) The deflected light pulse is detected with an EMCCD camera. (b) A detuned laser beam with a spatially varying intensity profile across the atoms creates a spatial phase gradient via the differential light shift. (c) Row sums of the CCD images from the deflected light pulse for different interaction times. Each curve is averaged over 5 runs and the background due to the coupling beam is subtracted. (d) Deflection angle $\beta$ as a function of the interaction time $t_{\text {int }}$ with the gradient beam. The blue line is a linear fit.

that imprinting a phase gradient on a stored-light state can change the direction of the restored pulse. This is similar to the work demonstrating deflection of light in a vapor cell by a magnetic gradient field [25] or by an inhomogeneous laser beam [26]. In our experiment, we first store a pulse in a MI with $\simeq 2.5 \times 10^{5}$ atoms, and a lattice depth of $30 E_{r}$ using the sequence described above $\left(\Omega_{c}=\right.$ $\left.2 \pi \times 4.3 \mathrm{MHz}, \Omega_{p}=2 \pi \times 3.8 \mathrm{MHz}\right)$. Before retrieving the pulse after $10 \mathrm{~ms}$ storage time, we shine in an additional $\sigma^{+}$polarized laser $\left(w_{0}=42(8) \mu \mathrm{m}\right)$, aligned $20(5) \mu \mathrm{m}$ away from the center of the atomic cloud [Fig. 4(b)]. The laser is red detuned from the $|2\rangle \leftrightarrow|3\rangle$ transition by $-20 \mathrm{GHz}$, which causes spatially inhomogeneous light shifts $\Delta_{1,2}(\vec{r})$ of the two ground state levels due to the Gaussian intensity profile. Shining in this laser for an 
interaction time $t_{\text {int }}$ induces a local dephasing between $|1\rangle$ and $|2\rangle$ of $\phi(\vec{r})=\left[\Delta_{1}(\vec{r})-\Delta_{2}(\vec{r})\right] t_{\text {int }} / \hbar$. In our experiment, the maximum laser intensity is $2.3(2) \mathrm{W} / \mathrm{cm}^{2}$, which produces a differential light shift of $\Delta_{1}-\Delta_{2}=$ $2 \pi \times 7.7 \mathrm{kHz}$ at the center of the atomic cloud. The interaction time $t_{\text {int }}$ is varied from 0 to $150 \mu \mathrm{s}$. The deflection angle is

$$
\beta \simeq \frac{\Delta k}{k_{p}} \quad \text { with } \quad \Delta k=\frac{d\left(\Delta_{1}-\Delta_{2}\right)}{d y} \frac{t_{\text {int }}}{\hbar},
$$

where $k_{p}=2 \pi / \lambda$ is the wavevector of the probe laser beam.

The deflected pulse is detected using an electron multiplying CCD (EMCCD, ANDOR iXon DV885), see Fig. $4(\mathrm{a})$. In order to reveal the deflection, the camera is placed out of the focal plane by translating the last lens before the camera. The detected signal on the EMCCD camera for $t_{\text {int }}=0 \mu$ s contains about $1.1 \times 10^{5}$ counts (corresponding to $3.4 \times 10^{3}$ photons). This signal was then summed along the $z$-direction and averaged over 5-12 runs for better visibility. To each of these integrated pulses we fit a 1D Gaussian and determine the position shift $\delta y$ of the deflected beam. From $\delta y$ and the camera position with respect to the focal plane, we determine the deflection angle $\beta$. The result is summarized in Fig. 4(d) together with a linear fit. The fitted slope $d \beta / d t_{\text {int }}=155(5) \mu \mathrm{rad} / \mu \mathrm{s}$ is close to the value of $232(46) \mu \mathrm{rad} / \mu$ s calculated from our experimental parameters. The error takes into account the uncertainties of the gradient beam power, waist and the alignment.

In summary we have demonstrated EIT, light storage and retrieval from an atomic Mott insulator. We have observed very long storage times of about $240 \mathrm{~ms}$, where the storage time is limited by heating from the lattice and by tunneling. We also demonstrated that a stored pulse can be controlled and redirected by imprinting a spatial phase gradient with a laser beam.

In the future, it would be interesting to extend this technique to more complex light fields in order to process and manipulate information stored in spin structures, which can then be analyzed by measuring the direction and shape of the retrieved pulse. In contrast two the usual manipulation of the spins by microwave radiation, EIT also allows the imprinting of elaborate phase structures generated by holograms such as images or vortices [27]. This could facilitate the study of farfrom equilibrium spinor gases, or allow the storage of a doubly charged $m=2$ vortex in the MI phase, where it is expected to be stable in contrast to a BEC [28]. Another interesting prospect is to use the MI as a genuine quantum memory to store and to retrieve single photons [17, 18]. By using an optimized geometry with a higher $\mathrm{OD}$, storage of an entire pulse or pulse sequence can be achieved. As an alternative to storing light pulses, one can also directly create an atomic superposition. Turning on the coupling field then leads to the creation of a probe field. We are currently exploring the use of such a created light pulse as a novel probe for classical or entangled atomic spin states in an optical lattice. Ultimately, the generation of such non-classical spin states and the direct mapping onto photonic states could lead to a new generation of non-classical light sources [20, 21].

We acknowledge support by DIP, DFG, EU (IP SCALA), AFOSR, and DARPA (OLE) and the Fulbright Association (J.D.T.). We thank A. V. Gorshkov for helpful discussions.
[1] L.-M. Duan, M. D. Lukin, J. I. Cirac, and P. Zoller, Nature 414, 413 (2001).

[2] M. D. Lukin, Rev. Mod. Phys. 75, 457 (2003).

[3] M. Mašalas and M. Fleischhauer Phys. Rev. A 69, 061801(R) (2004).

[4] M. Fleischhauer, A. Imamoglu, and J. P. Marangos, Rev. Mod. Phys. 77, 633 (2005).

[5] M. D. Lukin, S. F. Yelin, and M. Fleischhauer, Phys. Rev. Lett. 84, 4232 (2000); M. Fleischhauer and M. D. Lukin, Phys. Rev. Lett. 84, 5094 (2000).

[6] C. Liu, Z. Dutton, C. H. Behroozi, L. V. Hau, Nature 409, 490 (2001).

[7] D. F. Phillips et al., Phys. Rev. Lett. 86, 783 (2001).

[8] H. J. Kimble, Nature 453, 1023 (2008).

[9] T. Chanelière et al., Nature 438, 833 (2005).

[10] A. V. Turukhin et al., Phys. Rev. Lett. 88, 023602 (2002).

[11] M. M. Kash et al., Phys. Rev. Lett. 82, 5229 (1999).

[12] L. V. Hau, S. E. Harris, Z. Dutton, and C. H. Behroozi, Nature 397, 594 (1999).

[13] V. Ahufinger et al., Opt. Commun. 211, 159 (2002).

[14] J. J. Longdell, E. Fraval, M. J. Sellars, and N. B. Manson, Phys. Rev. Lett. 95, 063601 (2005).
[15] M. Klein, I. Novikova, D. F. Phillips, and R. L. Walsworth, J. Mod. Opt. 53, 2583 (2006).

[16] B. Julsgaard et al., Nature 432, 482 (2004).

[17] B. Zhao et al., Nature Phys. 5, 95 (2009).

[18] R. Zhao et al., Nature Phys. 5, 100 (2009).

[19] I. Bloch, J. Dalibard, and W. Zwerger, Rev. Mod. Phys. 80, 885 (2008).

[20] D. Porras and J. I. Cirac, Phys. Rev. A 78, 053816 (2008).

[21] L. H. Pedersen and K. Mølmer, Phys. Rev. A 79, 012320 (2009).

[22] D. M. Harber, H. J. Lewandowski, J. M. McGuirk, and E. A. Cornell, Phys. Rev. A 66, 053616 (2002).

[23] N. B. Phillips, A. V. Gorshkov, and I. Novikova, Phys. Rev. A 78, 023801 (2008).

[24] A. Widera et al., Phys. Rev. Lett. 92, 160406 (2004).

[25] L. Karpa and M. Weitz, Nature Phys. 2, 332 (2006).

[26] V. A. Sautenkov, H. Li, Y. V. Rostovtsev, M. O. Scully, arXiv:quant-ph/0701229v1 (2007).

[27] R. Pugatch et al., Phys. Rev. Lett. 98, 203601 (2007).

[28] Y. Shin et al., Phys. Rev. Lett. 93, 160406 (2004); J. A. M. Huhtamäki et al., Phys. Rev. Lett. 97, 110406 (2006). 